\title{
RINGS RELATED TO COMPLETELY O-SIMPLE SEMIGROUPS
}

\author{
D. B. MCALISTER
}

(Received 21 November 1968)

Communicated by G. B. Preston

A.H. Clifford ([2], [3]) has shown that all finite dimensional irreducible representations of a completely 0 -simple semigroup can be obtained as extensions of those of its maximal subgroups. Lallement and Petrich, [7], have given an alternative method for constructing the irreducible representations of a finite 0 -simple semigroup from its Schutzenberger representation ([13]). Using the form which they obtain for the irreducible representations of a finite 0 -simple semigroup $S=\mathscr{M}^{0}(G ; m, n ; P)$, Lallement and Petrich show that

$$
\operatorname{Rad} \mathscr{M}=\{X \in \mathscr{M}: P X P \text { is over } \operatorname{Rad} \Phi(G)\}
$$

where $\mathscr{M}$ is the Munn algebra isomorphic to the (contracted) algebra $\Phi(S)$ of $S$ over $\Phi$.

In the same paper, necessary and sufficient conditions are given for the existence of an isomorphism

$$
\Phi(S) / \operatorname{Rad} \Phi(S) \approx(\Phi(G) / \operatorname{Rad} \Phi(G))_{t}
$$

when $\Phi$ is algebraically closed and $t$ is the invertibility rank of $P$. However the general problem of determining the structure of $\Phi(S) / \operatorname{Rad} \Phi(S)$ is not solved there nor is the case of infinite completely 0 -simple semigroups considered. The main object of this paper is to determine the radical of $\Phi(S)$ when $S$ is an arbitrary completely 0 -simple semigroup and also to obtain a representation for $\Phi(S) / \operatorname{Rad} \Phi(S)$ as a subdirect sum of computable primitive rings.

The representation theory developed in [2], [3] depends strongly on the fact that the representations under consideration are finite dimensional and it is not adequate to deal with the infinite dimensional case. In [9] we have described a variant of Clifford's theory which determines all representations of $S \approx \mathscr{M}^{0}(G ; I, \Lambda ; P)$, by endomorphisms of $\mathfrak{R}$-modules, in terms of proper representations of $G$. This representation theory was developed for a class of rings which we call Munn rings. These rings are the infinite dimensional analogs of the Munn algebras considered in [11], Section 4; thus the algebra of any completely 0 -simple semigroup is a Munn ring.

The results of [9] provide the tools with which to investigate the ideal struc- 
ture of a Munn ring and to find its primitive homomorphic images. Throughout this paper, we shall assume familiarity with the results and terminology of [9].

In Section 1 , the ideal structure of a Munn ring $\mathscr{M}=\mathscr{M}(\mathfrak{A} ; I, \Lambda ; P)$ is investigated and the primitive ideals of $\mathscr{M}$ are determined in terms of the primitive ideals of $\mathfrak{A}$. It is shown that

(Theorem 1.6).

$$
\operatorname{Rad} \mathscr{M}=\{X \in \mathscr{M}: P X P \text { is over } \operatorname{Rad} \mathfrak{U}\}
$$

In the second section, the primitive homomorphic images of the Munn ring $\mathscr{M}$ are determined (Theorem 2.3). From this theorem, a representation of $\mathscr{H} / \operatorname{Rad} \mathscr{M}$ as a subdirect sum of primitive rings, is evident. In the case when $\mathscr{H}$ satisfies the descending chain condition (d.c.c.) on right ideals. $\mathfrak{A}$ satisfies the d.d.c. on right ideals and $I$ is finite. Further an exact description of $\mathscr{M} / \operatorname{Rad} \mathscr{M}$ can be given (Theorem 2.5).

Section 3 is concerned with the special case which was considered by Lallement and Petrich ([7], Theorem 3.6). Using the structure given by Theorem 2.5 for $\mathscr{M} / \operatorname{Rad} \mathscr{M}$ when $\mathscr{M}=\mathscr{M}(\mathfrak{A} ; m, n ; P)$ obeys the d.c.c. on right ideals, we give necessary and sufficient conditions for the existence of an isomorphism

$$
\mathscr{H} / \operatorname{Rad} \mathscr{M} \approx(\mathfrak{U} / \operatorname{Rad} \mathfrak{A})_{t}
$$

where $t$ is the invertibility rank of $P$; (Theorem 3.4). Unfortunately, the existence of such an isomorphism does not characterise $\mathscr{M}$, even in the case when $t=1$. In order to provide such a characterisation of $\mathscr{M}$, one has to consider the basic radical of $\mathscr{M}$ which is

$$
B(\mathscr{H})=\{X \in \mathscr{M}: P X P=0\} .
$$

Theorem 3.11 gives necessary and sufficient conditions for the existence of an isomorphism

$$
\mathscr{M} / B(\mathscr{H}) \approx(\mathfrak{A})_{t}
$$

again where $t=$ invertibility rank $P$. In the presence of such an isomorphism $\mathscr{M}$ itself is characterised up to isomorphism. As a corollary to Theorem 3.11, we show that a finite 0 -simple semigroup $S$ is a regular rectangular semigroup with zero adjoined if and only if

$$
\Phi(S) / B(\Phi(S)) \approx \Phi(G)
$$

Because the algebra of a finite 0 -simple semigroup is a Munn ring which obeys the d.c.c. on right ideals, the results obtained in previous sections apply to these semigroups. In fact, Theorem 2.5 can be used to describe the algebra of an arbitrary finite semigroup modulo its radical. This is done in Section 4.

\section{The ideals of a Munn ring}

Definition 1.1. An ideal $N$ of a Munn ring $\mathscr{M}$ is basic if it is the kernel of a basic representation of $\mathscr{M}$. 
Proposition 1.2. An ideal N of a Munn ring $\mathscr{M}$ is basic if and only if $\mathscr{M}$ a $\mathscr{M} \subseteq N$ implies $a \in N$. If this is the case then the representation of $\mathscr{M}$ on the cosets of $\mathscr{M} / N$ is basic with kernel $N$.

The intersection of basic ideals is basic and hence, for any ideal $N$ of $\mathscr{M}$, there is a minimum basic ideal which contains $N$; this is

$$
B(N)=\{a \in \mathscr{M}: \mathscr{M} a \mathscr{M} \subseteq N\} .
$$

In particular, the intersection of all basic ideals is basic and is

$$
B(0)=\{a \in \mathscr{M}: \mathscr{M} a \mathscr{M}=0\} .
$$

Proof. Suppose that $N$ is basic and let $a \in \mathscr{M}$ be such that $\mathscr{M}$ a $\mathscr{M} \subseteq N$. Let $\Gamma: \mathscr{M} \rightarrow \operatorname{Hom}(C, C)$ be a basic representation of $\mathscr{M}$, over a ring $\mathfrak{R}$ with identity, which has kernel $N$. For each subset $U$ of $C$ denote by $[U]$ the $\Re$-submodule of $C$ generated by $U$; since $\Gamma$ is basic, $[C \Gamma(\mathscr{M})]=C$.

Since $\mathscr{M} a \mathscr{M} \subseteq N,[C \Gamma(\mathscr{M} a \mathscr{M})]=0$. Thus $C \Gamma(x a y)=0$ for all $x, y \in \mathscr{M}$. This implies $[C \Gamma(\mathscr{M}) \Gamma(a y)]=0$ for each $y \in \mathscr{M}$. Therefore, since $\Gamma$ is basic, $C \Gamma(a)$ is a submodule or $C$ which is contained in $N(\Gamma)$ and hence is zero. This means $\Gamma(a)=0$ and so, since $N=\operatorname{Ker} \Gamma, a \in N$.

Conversely, suppose $\mathscr{M} a \mathscr{M} \subseteq N$ implies $a \in N$ and consider the representation $\Gamma$ of $\mathscr{M}$ on $\mathscr{M} / N$. Then

$$
I(\Gamma)=\text { subgroup of } \mathscr{M} / N \text { generated by the cosets } N+x a
$$

where $a, x \in \mathscr{M}$;

and

$$
N(\Gamma)=\{N+x: x a \in N \text { for all } a \in \mathscr{M}\} ;
$$

$\operatorname{Ker} \Gamma=\{a \in \mathscr{M}: x a \in N$ for all $x \in \mathscr{M}\}$.

The fact that $\mathscr{M} a \mathscr{M} \subseteq N$ implies $a \in N$ shows immediately that $N(\Gamma)=N$, the zero of $\mathscr{M} / N$ and $\operatorname{Ker} \Gamma=N$. Further since, by the proof of [9], Corollary $1.6, \mathscr{M}$ is generated as a group by $\mathscr{M}^{2}$,

$$
I(\Gamma)=\text { subgroup generated by the cosets in } N+\mathscr{M}^{2}=\mathscr{M} / N .
$$

That the intersection of basic ideals is basic follows because, as is easily seen, the direct sum of basic representations is basic. To prove the final two assertions it is thus only necessary to prove that

$$
B(N)=\{a: \mathscr{M} a \mathscr{K} \subseteq N\}
$$

is the smallest basic ideal containing $N$. From the proof of the characterisation already obtained, it follows that $B(N)$ is certainly contained in any basic ideal which contains $N$. Hence it suffices to prove that $B(N)$ is basic. To do this, we use the characterisation obtained above.

Suppose $\mathscr{M} a \mathscr{M} \subseteq B(N)$; then $\mathscr{M}^{2} a \mathscr{H}^{2} \subseteq N$ and so, since $\mathscr{M}$ is generated as a subgroup by $\mathscr{M}^{2}, \mathscr{M} a \mathscr{M} \subseteq N$. Thus $a \in B(N)$ and $B(N)$ is basic. 
Although Proposition 1.2 gives a characterisation of the basic ideals of a Munn ring, it does not give very much information about their structure. Much stronger results can be obtained; in fact, the basic ideals of $\mathscr{M}(\mathfrak{Q} ; 1, \Lambda ; P)$ can be described precisely in terms of the ideals of $\mathfrak{A}$. This is done in Theorem 1.4.

Let $\mathscr{M}=\mathscr{M}(\mathfrak{A} ; I, \Lambda ; P)$ be a Munn ring and let $M$ be an ideal of $\mathscr{M}$. For each $(i, \lambda) \in I \times \Lambda$ such that $p_{\lambda i}$ is invertible, we obtain an ideal $M_{i \lambda}$ of $\mathfrak{A}$ as follows:

$$
M_{i \lambda}=\{a \in \mathfrak{A}:(a ; i, \lambda) \in M\} .
$$

LEMma 1.3. If $(i, \lambda),(j, \mu) \in I \times \Lambda$ are such that $p_{\lambda i}$ and $p_{\mu j}$ are invertible then $M_{i \lambda}=M_{j \mu}$.

Proof. If $a \in M_{i \lambda}$ then $(a ; i, \lambda) \in M$ and thus

$$
(a ; j, \mu)=\left(p_{\lambda i}^{-1} ; j, \lambda\right)(a ; i, \lambda)\left(p_{\lambda i}^{-1} ; i, \mu\right)
$$

also belongs to $M$. Hence $M_{i \lambda} \subseteq M_{j \mu}$ and, by symmetry, $M_{j \mu} \subseteq M_{i \lambda}$. This proves the result.

As a consequence of Lemma 1.3, it makes sense to speak of the ideal $I(M)$ of $\mathfrak{A}$ induced by an ideal $M$ of $\mathscr{M}$. On the other hand, given any ideal $N$ of $\mathfrak{A}$, the set

is an ideal of $\mathscr{M}$.

$$
B(N)=\{X \in \mathscr{M}: P X P \text { is over } N\}
$$

In the following theorem, let $\mathscr{L}(\mathfrak{H}), \mathscr{L}(\mathscr{M})$ denote, respectively, the lattices of ideals of $\mathfrak{A}$ and $\mathscr{M}$. Further, let $\mathscr{B}(\mathscr{M})$ denote the $\wedge$-subsemilattice of $\mathscr{L}(\mathscr{M})$ which consists of the basic ideals of $\mathscr{M}$.

THEOREM 1.4. The mapping $I: \mathscr{L}(\mathscr{M}) \rightarrow \mathscr{L}(\mathfrak{A})$, which associates, with each ideal $M$ of $\mathscr{M}$, the induced ideal $I(M)$ of $\mathfrak{A}$, is a complete lattice homomorphism of $\mathscr{L}(\mathscr{M})$ onto $\mathscr{L}(\mathfrak{H})$. The mapping $B: \mathscr{L}(\mathfrak{H}) \rightarrow \mathscr{L}(\mathscr{M})$ is a $\wedge$-complete semilattice homomorphism of $\mathscr{L}(\mathfrak{U})$ into $\mathscr{L}(\mathscr{M})$ whose image is $\mathscr{B}(\mathscr{M})$. Further, $I \circ B$ is the identity mapping on $\mathscr{L}(\mathfrak{U})$ and, for each $M \in \mathscr{L}(\mathfrak{U}), B(M)$ is the maximum ideal $N$ of $\mathscr{M}$ such that $I(N)=M$.

Proof. Let $\left\{M_{k}: k \in K\right\}$ be a set of ideals of $\mathscr{M}$ and let $(i, \lambda) \in I \times \Lambda$ be such that $p_{\lambda i}$ is invertible. Then

$$
a \in I\left(\cap\left\{M_{k}: k \in K\right\}\right) \text { if and only if }(a ; i, \lambda) \in \cap\left\{M_{k}: k \in K\right\}
$$

which occurs if and only if $a \in \cap\left\{I\left(M_{k}\right): k \in K\right\}$. Hence

$$
I\left(\cap\left\{M_{k}: k \in K\right\}\right)=\cap\left\{I\left(M_{k}\right): k \in K\right\} .
$$

On the other hand, it is easy to see that

$$
\vee\left\{I\left(M_{k}\right): k \in K\right\} \subseteq I\left(\vee\left\{M_{k}: k \in K\right\}\right) .
$$

Hence, when we prove the converse inclusion, we will have shown that $I$ is a complete lattice homomorphism. 
Let $X \in \mathscr{H}$; then

$$
\left(p_{\lambda i}^{-1}: i, \lambda\right) X\left(p_{\lambda i}^{-1} ; i, \lambda\right)=(b ; i, \lambda)
$$

for some $b \in \mathfrak{A}$. Hence, if $(a ; i, \lambda) \in \vee\left\{M_{k}: k \in K\right\}$, then

$$
\begin{aligned}
(a ; i, \lambda) & =\left(p_{\lambda i}^{-1} ; i, \lambda\right)(a ; i, \lambda)\left(p_{\lambda i}^{-1} ; i, \lambda\right) \\
& =\left(b_{1} ; i, \lambda\right)+\cdots+\left(b_{n} ; i, \lambda\right)
\end{aligned}
$$

where $\left(b_{j} ; i, \lambda\right) \in \cup\left\{M_{k}: k \in K\right\}, 1 \leqq j \leqq n$.

Hence $a=b_{1}+\cdots+b_{n} \in \vee\left\{I\left(M_{k}\right): k \in K\right\}$. This proves

$$
I\left(\vee\left\{M_{k}: k \in K\right\}\right) \subseteq \vee\left\{I\left(M_{k}\right): k \in K\right\}
$$

so that we have equality.

Now consider $B$ and this time let $\left\{M_{k}: k \in K\right\}$ denote a set of ideals of $\mathfrak{A}$. Then $X \in B\left(\cap\left\{M_{k}: k \in K\right\}\right)$ if and only if $P X P$ is over $\cap\left\{M_{k}: k \in K\right\}$. But this is possible if and only if $P X P$ is over $M_{k}$ for each $k \in K$. Hence

$$
B\left(\cap\left\{M_{k}: k \in K\right\}\right)=\cap\left\{B\left(M_{k}\right): k \in K\right\}
$$

and so $B$ is a $\wedge$-complete semilattice homomorphism.

Next we show that $I \circ B$ is the identity on $\mathscr{L}(\mathfrak{A})$; this proves that $I$ is onto as claimed in the statement of the theorem. Let $M$ be an ideal of $\mathfrak{A}$ and let $\gamma: \mathfrak{A} \rightarrow$ Hom $(\mathfrak{U} / M, \mathfrak{A} / M)$ be the representation of $\mathfrak{A}$ on $\mathfrak{A} / M$; since $\mathfrak{U}$ has an identity, $\gamma$ is proper. Let $Q, R$ be epic and monic respectively such that $Q R=\gamma(P)$. Then, by [9], Theorem $2.7, \Gamma=[\gamma ; Q, R]$ is a basic representation of $\mathscr{M}$ with kernel $B(M)$; hence $B(M)$ is basic. Further, since the representation of $\mathfrak{U}$ induced by $\Gamma$ is equivalent to $\gamma, I(B(M))=M$. Thus $I \circ B$ is the identity on $\mathscr{L}(\mathfrak{A})$.

The fact that $B(M)$ is basic for each $M \in \mathscr{L}(\mathfrak{U})$ shows that $\operatorname{Im} B \subseteq \mathscr{B}(\mathscr{M})$. On the other hand, [9], Theorem 2.7 shows that each basic ideal of $\mathscr{M}$ is of the form $B(M)$ for some $M \in \mathscr{L}(\mathfrak{U})$. Hence $\operatorname{Im} B=\mathscr{B}(\mathscr{M})$.

Finally, let $N$ be any ideal of $\mathscr{M}$ with $M=I(N)$ and denote by $\mathscr{M}^{1}$ the ring obtained by adjoining an identity to $\mathscr{M}$. Then $N$ is an ideal of $\mathscr{H}^{1}$ and the mapping $\Gamma: \mathscr{M} \rightarrow \operatorname{Hom}\left(\mathscr{M}^{1} / N, \mathscr{M}^{1} / N\right)$ defined by

$$
(N+x) \Gamma(X)=N+x X,
$$

for each $x \in \mathscr{M}^{1}, X \in \mathscr{M}$, is a representation of $\mathscr{M}$ with kernel $N$. This representation is of the form

$$
\Gamma(X)=R \gamma(X) Q
$$

where $\gamma$ is a representation of $\mathfrak{A}$ with kernel $M$ and $Q R=\gamma(P)$. Then $X \in N$ implies $R \gamma(X) Q=0$ and thus $\gamma(P X P)=0$. Therefore $N \subseteq B(M)$.

The following corollary generalises the well known result for ideals in matrix rings ([10], Theorem 2.24); a slight adaptation of the argument in [10] also suffices to prove the more general result. 
COROLLARY 1.5. Let $\mathfrak{A}$ be a ring with identity and let I be a set. Then the ideals of the ring $\mathscr{M}$ of all finitely non-zero $I \times I$ matrices over $\mathfrak{A}$ are the sets of the form

where $M$ is an ideal of $\mathfrak{A}$.

$$
\{X \in \mathscr{M}: X \text { is over } M\}
$$

Proof. $\mathscr{M} \approx \mathscr{M}(\mathfrak{H} ; I, I ; \Delta)$ where $\Delta$ is the $I \times I$ identity matrix. If $N$ is any ideal of $\mathscr{M}$ with $I(N)=M$ then there is a representation $\gamma: \mathfrak{U} \rightarrow \operatorname{Hom}(A, A)$ and $Q:{ }^{I} A \rightarrow C, R: C \rightarrow A^{I}$ such that $Q R=\gamma(\Delta)$ and further

$$
N=\{X \in \mathscr{M}: R \gamma(X) Q=0\} .
$$

Thus $X \in N$ implies $\gamma(\Delta X \Delta)=0$.

Now $\gamma(\Delta X \Delta)$ is the morphism ${ }^{I} A \rightarrow A^{I}$ determined by the $I \times I$ matrix $\gamma(X)$. Hence $X \in N$ implies $\gamma(X)=0$ while, on the other hand, if $\gamma(X)=0$ then $X \in N$. Since $M=\operatorname{Ker} \gamma$, it follows that

$$
N=\{X \in \mathscr{M}: X \text { is over } M\} .
$$

THeOREM 1.6. Let $\mathscr{M}=\mathscr{M}(\mathfrak{A} ; I, \Lambda ; P)$ be a Munn ring. Then the basic ideals of $\mathscr{M}$ are the ideals

$$
B(M)=\{X \in \mathscr{M}: P X P \text { is over } M\}
$$

where $M$ is an ideal of $\mathfrak{A}$.

$$
\begin{aligned}
& B(M) \text { is maximal if and only if } M \text { is maximal; } \\
& B(M) \text { is primitive if and only if } M \text { is primitive. }
\end{aligned}
$$

Further, the radical of $\mathscr{M}$ is

$$
\operatorname{Rad} \mathscr{M}=\{X \in \mathscr{M}: P X P \text { is over } \operatorname{Rad} \mathfrak{A}\} .
$$

Proof. That the basic ideals are of the form $B(M)$ above is merely a restatement of part of Theorem 1.4. From Theorem 1.4, any maximal ideal is basic and so of the form $B(M)$ for some $M \in \mathscr{L}(\mathfrak{Q})$. But $M \subseteq N$ implies $B(M) \subseteq B(N)$ so that $M$ must be maximal in $\mathscr{L}(\mathfrak{H})$. On the other hand, if $M$ is a maximal ideal of $\mathfrak{U}$ and $B(M) \subseteq N$ then $M=I \circ B(M) \subseteq I(N)$. Hence $M=I(N)$ so that $B(M)=$ $B(I(N)) \supseteq N$. Hence $B(M)$ is maximal.

An ideal $N$ of $\mathscr{M}$ is primitive if and only it it is the kernel of an irreducible representation of $\mathscr{M}$ over the integers. Therefore, by [9], Theorem 3.7, $N$ is primitive if and only if $N=B(M)$ where $M$ is the kernel of an irreducible representation of $\mathfrak{A}$ over the integers. Thus $N$ is primitive if and only if $N=B(M)$ where $M$ is a primitive ideal of $\mathfrak{A}$.

Finally, the radical of $\mathscr{M}$ is the intersection of its primitive ideals. Hence

$$
\begin{aligned}
\operatorname{Rad} \mathscr{M} & =\cap\{B(M): M \text { is a primitive ideal of } \mathfrak{A}\} \\
& =B(\cap\{M: M \text { is primitive in } \mathfrak{A}\}) \\
& =B(\operatorname{Rad} \mathfrak{A}) .
\end{aligned}
$$


Remark 1.7. Lallement and Petrich [7] have shown that, if $\mathscr{M}$ is the algebra of a finite 0 -simple semigroup $\mathscr{M}^{0}(G ; I, \Lambda ; P)$, over a field $\Phi$, then

$$
\operatorname{Rad} \mathscr{M}=\{X \in \mathscr{M}: P X P \text { is over } \operatorname{Rad} \Phi(G)\} .
$$

Brown [1] has considered algebras which he called generalised matrix algebras. These are Munn rings of the form $\mathscr{M}(\Phi ; m, n ; P)$ where $\Phi$ is a field of characteristic zero and Brown shows that

$$
\operatorname{Rad} \mathscr{M}=\{X \in \mathscr{M}: P X P=0\} .
$$

\section{The primitive homomorphic images}

In this section, we use the Jacobson Density Theorem [6] to characterise the primitive homomorphic images of a Munn ring. We shall adhere to the terminology and notation used in [10], Chapter 5 in connection with the density theorem, except that we require all modules to be left modules.

LEMMA 2.1. Let $\mathfrak{A}$ be a dense ring of linear transformations of a vector space $A$ over a division ring $D$. If $n$ is a positive integer, then the ring $\mathfrak{A}_{n}$ of $n \times n$ matrices over $\mathfrak{A}$ is a dense ring of linear transformations of the vector space $A^{n}={ }^{n} A$ over $D$.

ProOF. Certainly $\mathfrak{A}_{n}$ acts as a singly transitive ring of endomorphisms of ${ }^{n} A$ and so is irreducible. By the density theorem $\mathfrak{A}_{n}$ is therefore a dense ring of linear transformations of ${ }^{n} A$ considered as a vector space over the opposite ring to the centraliser of $\mathfrak{A}_{n}$.

Easy calculation shows that the centraliser of $\mathfrak{A}_{n}$ is isomorphic to the opposite ring of $D$ and that the scalar multiplication induced on ${ }^{n} A$ coincides with the ordinary multiplication by scalars in $D$. Hence we have the result.

Lemma 2.2. Let $A$ be a vector space over a division ring $D$ and let $I$ be an infinite set. If $f^{1}, \cdots, f^{n}$ are linearly independent in the vector space $A^{x}$ over $D$ then there exist $i_{1}, \cdots, i_{n} \in I$ such that $g^{1}, \cdots, g^{n}$, defined below, are linearly independent; $g^{j}$ is given by

$$
g_{i}^{j}=g^{j} \pi_{i}=\left\{\begin{array}{cl}
f_{i}^{j}=f^{j} \pi_{i} & \text { if } i=i_{1}, \cdots, i_{n} \\
0 & \text { otherwise }
\end{array}\right.
$$

for $j=1,2, \cdots, n$.

Proof. Pick $i_{1} \in I$ such that $f_{i_{1}}^{1} \neq 0$. If $f_{i_{1}}^{1}, \cdots f_{i_{1}}^{n}$ are linearly independent we are done; we need only pick any $n-1$ other elements $i_{2}, \cdots, i_{n}$ of $I$. If not, we can manipulate $f^{1} \cdots f^{n}$ so that $f_{i_{1}}^{1}, \cdots f_{i_{1}}^{s}$ are linearly independent and $f_{i_{1}}^{s+1}, \cdots, f_{i_{1}}^{n}$ are all zero without destroying the linear independence of $f^{1}, \cdots, f^{n}$. Since the $n-s$ vectors $f^{s+1}, \cdots, f^{n}$ are linearly independent, there exist, by means of an obvious induction $i_{s+1}, \cdots, i_{n}$ such that $k^{s+1}, \cdots, k^{n}$, defined by 


$$
k_{i}^{j}= \begin{cases}f_{i}^{j} & i=i_{s+1}, \cdots, i_{n}, \\ 0 & \text { otherwise }\end{cases}
$$

$s+1 \leqq j \leqq n$ are linearly independent. Pick $i_{2}, \cdots, i_{s} \in I$ distinct from each other and from $i_{1}, i_{s+1}, \cdots, i_{n}$ and define $g^{1}, \cdots, g^{n}$ as in the statement of the lemima. Then $g^{1}, \cdots, g^{n}$ are linearly independent.

THEOREM 2.3. Let $\mathscr{M}=\mathscr{M}(\mathfrak{A} ; I, A ; P)$ be a Munn ring and let $M$ be a primitive ideal of $\mathscr{M}$. Let $\gamma: \mathfrak{A} \rightarrow \operatorname{Hom}(A, A)$ be a morphism of $\mathfrak{A}$, with image a dense ring of linear transformations of a vector space $A$ over a division ring $D$, and suppose that $M=\operatorname{Ker} \gamma$.

Let $C=A / \operatorname{Ker} \gamma(P)$, where $\gamma(P)$ is regarded as a morphism ${ }^{A} A \rightarrow A^{I}$ of vector spaces over $D$. Then $\mathscr{M} / B(M)$ is isomorphic to a dense ring of linear transformations of the vector space $C$ over $D$.

Proof. Let $Q:{ }^{A} A \rightarrow C$ and $R: C \rightarrow A^{I}$ be morphisms of vector spaces over $D$ such that $Q R=\gamma(P)$ with $Q$ epic and $R$ monic. Then $\Gamma: \mathscr{M} \rightarrow \operatorname{Hom}(C, C)$, where $\Gamma=[\gamma ; Q, R]$, is, by [9] Theorem 3.7, an irreducible representation of $\mathscr{M}$ over $D$. Further $\Gamma(\mathscr{M}) \approx \mathscr{M} / B(M)$.

To show that $\Gamma(\mathscr{M})$ is a dense ring of linear transformations of $C$, it suffices to prove that $\Gamma(\mathscr{M})$ is singly and doubly transitive on $C$; [10], Theorem 5.47 . If $C$ does not have dimension one over $D$, it in fact suffices to show that $\Gamma(\mathscr{M})$ is doubly transitive on $C$. We shall assume that $\operatorname{dim} C \geqq 2$ and prove that $\Gamma(\mathscr{M})$ is doubly transitive on $C$; the proof in the case when $\operatorname{dim} C=1$ is essentially similar.

Let $\left(c_{1}, c_{2}\right),\left(d_{1}, d\right)$ be pairs of linearly independent elements of $C$. Then there are linearly independent elements $e^{1}, e^{2}$ in $A$ such that $e^{i} Q=d_{i}, i=1,2$. Further, since $R$ is monic, $f^{1}=c_{1} R$ and $f^{2}=c_{2} R$ are linearly independent. Hence it suffices to find $X \in \mathscr{H}$ such that $f^{i} \gamma(X)=e^{i}, i=1,2$.

Since $f^{1}, f^{2}$ are linearly independent, there exist $u, v \in I$ such that $g^{1}, g^{2}$ defined by

$$
g_{k}^{i}=\left\{\begin{array}{cl}
f_{k}^{i} & \text { if } k=u, v \\
0 & \text { otherwise }
\end{array}\right.
$$

are linearly independent. Suppose that, for some $X \in \mathscr{M}$,

and define $Y \in \mathscr{M}$ by

$$
g^{i} \gamma(X)=e^{i} \quad i=1,2
$$

$$
Y_{h \lambda}=\left\{\begin{array}{cl}
X_{h, \lambda} & \text { if } h=u, v \\
0 & \text { otherwise }
\end{array}\right.
$$

Then

$$
f^{i} \gamma(Y)=g^{i} \gamma(Y)=g^{i} \gamma(X)=e^{i} \quad i=1,2
$$

Thus it suffices to find $X \in \mathscr{M}$ such that

$$
g^{i} \gamma(X)=e^{i} \quad i=1,2
$$


Now $e^{1}, e^{2}$ have non-zero components belonging to at most a finite number of the $A$; say $m$. Let $n=\max (m, 2)$. Then, as a final reduction of the problem, it suffices to show that the $n \times n$ matrices over $\gamma(\mathfrak{U})$ are a dense ring of linear transformations of the vector space ${ }^{n} A=A^{n}$ over $D$. That this is the case, is ensured by Lemma 2.1 .

Theorem 2.3 can be applied to give a complete description of the algebra of a Munn ring $\mathscr{M}$, which obeys the descending chain conditions on right ideals, modulo its radical. Thus, in particular, we can give a complete description of the algebra of a finite 0 -simple semigroup modulo its radical. This answers a question raised by Lallement and Petrich [7].

Proposition 2.4. Let $\mathscr{M}=\mathscr{M}(\mathfrak{A} ; I, A ; P)$ be a Munn ring. If $\mathscr{M}$ obeys the descending chain condition for right ideals, then $\mathfrak{A}$ obeys the descending chain condition for right ideals and I is finite.

Proof. For each right ideal $M$ of $\mathfrak{A}$ define

$$
R(M)=\{X \in \mathscr{M}: X P \text { is over } M\} .
$$

Then $R(M)$ is a right ideal of $\mathscr{M}$ and further $\left(a p_{\lambda i}^{-1} ; i, \lambda\right) \in R(M)$ if and only if $a \in M$, for each $(i, \lambda) \in I \times A$ such that $p_{\lambda i}$ is invertible. Hence $M \subset N$ implies $R(M) \subset R(N)$. This shows that if $\mathscr{M}$ obeys the d.c.c. on right ideals, so does $\mathscr{A}$.

For each subset $I^{\prime}$ of $I$, define

$$
\mathscr{M}\left(I^{\prime}\right)=\left\{X \in \mathscr{M}: X_{j \lambda}=0 \text { if } j \notin I^{\prime}\right\} .
$$

Then $\mathscr{M}\left(I^{\prime}\right)$ is a right ideal of $\mathscr{M}$ and $I^{\prime \prime} \subset I^{\prime}$ implies $\mathscr{M}\left(I^{\prime \prime}\right) \subseteq \mathscr{M}\left(I^{\prime}\right)$. Hence, if $\mathscr{M}$ obeys the d.c.c. on right ideals, $I$ is finite.

Let $\mathfrak{A}$ be a ring which obeys the d.c.c. on right ideals such that $\mathfrak{A}$ is generated as a group by $\mathfrak{A}^{2}$. Then each proper primitive ideal of $\mathfrak{A}$ is maximal. Further $\mathfrak{A}$ has only a finite number of maximal ideals, $M_{1}, \cdots, M_{s}$ say, and

$$
\mathfrak{U} / \operatorname{Rad} \mathfrak{U} \approx \mathfrak{H} / M_{1} \oplus \cdots \oplus \mathfrak{U} / M_{s} .
$$

Each $\mathfrak{A} / M_{i}$ is isomorphic to the ring of linear transformations of a finite dimensional vector space $V_{i}$ over a division ring $D_{i}$. Further $D_{i}$ and the dimension $s_{i}$ of $V_{i}$ are uniquely determined by $M_{i}$ so that $\mathfrak{A} / M_{i} \approx\left(D_{i}\right)_{s i}$ for a unique division ring $D_{i}$ and positive integer $s_{i}$. If $\gamma: \mathfrak{A} \rightarrow \operatorname{Hom}(A, A)$ is a ring morphism of onto a dense ring of linear transformations of a vector space $A$ over a division ring $D$, then $\gamma$ is onto $\operatorname{Hom}(A, A), D=D_{i}$ and $A=V_{i}$ where $\operatorname{Ker} \gamma=M_{i}$. The results described above follow from the Wedderburn-Artin Theorem, [10], Theorem 5.59 and the isomorphism theorem of [7], page 45 .

Suppose now that, as well as satisfying the d.c.c. for right ideals, $\mathfrak{A}$ has an identity and let $\Lambda$ be a set and $n$ a positive integer; let $P:{ }^{A} \mathscr{A} \rightarrow \mathfrak{U}^{n}$ be a morphism of $\mathfrak{U}$-modules. Then, for each morphism $\gamma_{i}: \mathfrak{A} \rightarrow\left(D_{i}\right)_{s_{i}}$ with kernel $M_{i}, \gamma_{i}(P)$ has finite rank $\left(=\operatorname{dim} \operatorname{Im} \gamma_{l}(P)\right)$ over $D_{i}$. 
Since $D_{i}, s_{i}$ are uniquely determined up to isomorphism and since $\gamma_{i}(P)$ is defined in a natural manner from $\gamma_{i}$, it follows that rank $\gamma_{i}(P)$ depends only on $M_{i}$. We call this rank the $M_{i}$-rank of $P$ and denote it by $t_{i}$.

TheOREM 2.5. Let $\mathscr{M}=\mathscr{M}(\mathfrak{A} ; I, \Lambda ; P)$ be a Munn ring which obeys the d.c.c. on right ideals. Then $\mathfrak{A}$ obeys the d.c.c. on right ideals and I is finite.

Suppose that $M_{1}, \cdots, M_{n}$ are the distinct maximal ideals of $\mathfrak{A}$ and that these have associated division rings $D_{1}, \cdots, D_{n}$. Then

and

$$
\operatorname{Rad} \mathscr{M}=\{X \in \mathscr{M}: P X P \text { is over } \operatorname{Rad} \mathfrak{U}\}
$$

$$
\mathscr{M} / \operatorname{Rad} \mathscr{M} \approx\left(D_{1}\right)_{t_{1}} \oplus \cdots \oplus\left(D_{n}\right)_{t_{n}} .
$$

Proof. Only the statement about $\mathscr{M} / \operatorname{Rad} \mathscr{M}$ still has to be proved. By the Wedderburn Artin theorem, $\mathscr{M} / \operatorname{Rad} \mathscr{M}$ is isomorphic to the direct sum of the rings $\mathscr{M} \mid N_{i}, i=1,2, \cdots, k$, where $N_{i}, i=1,2, \cdots, k$ are the distinct maximal ideals of $\mathscr{M}$. By Theorem 1.6, the maximal ideals are the ideals $B\left(M_{i}\right), i=1,2, \cdots n$.

Let $D_{i}$ be the division ring associated with $M_{i}$ and let $\gamma_{i}$ be the homomorphism of $\mathfrak{A}$ onto $\operatorname{Hom}(A, A)$, where $A$ is a vector spcae over $D_{i}$, with kernel $M_{i}$. Let $|I|=m$; then $\gamma_{i}(P)$ is a morphism ${ }^{A} A \rightarrow A^{m}$ of vector spaces over $D_{i}$. Hence $A / \operatorname{Ker}_{i}(P) \approx{ }^{t_{i}} D_{i}=D_{i}^{t_{i}}$ where $t_{i} \leqq m \operatorname{dim} A$; by definition, $t_{i}$ is the $M_{i}$-rank of $P$.

By Theorem 2.3, $\mathscr{M} / B\left(M_{i}\right)$ is (isomorphic to) a dense ring of the vector space ${ }^{A} A / \operatorname{Ker} \gamma_{i}(P)$, over $D_{i}$. Since ${ }^{A} A / \operatorname{Ker} \gamma_{i}(P)$ is finite dimensional, this means

$$
\mathscr{M} / B\left(M_{i}\right) \approx \operatorname{Hom}\left({ }^{A} A / \operatorname{Ker} \gamma_{i}(P),{ }^{A} A / \operatorname{Ker} \gamma_{i}(P)\right) \approx \operatorname{Hom}\left(D_{i}^{t_{i}}, D_{i}^{t_{i}}\right) \approx\left(D_{i}\right)_{t_{i}} .
$$

Hence we have the result.

As an immediate corollary to Theorem 2.5 , we can obtain the structure of the algebra $\mathscr{M}$ of a finite 0 -simple semigroup $\mathscr{M}^{0}(G ; m, n ; P)$, over a field $\Phi$, modulo its radical. In the case in which the field $\Phi$ is algebraically closed, or the irreducible representations of the structure group are one dimensional over $\Phi$, the determination of $\mathscr{M} / \operatorname{Rad} \mathscr{M}$ is particularly easy; it is only necessary to calculate rank $\gamma(P)$, over $\Phi$, for each irreducible representation $\gamma$ of $G$.

EXAmPLE 2.6. Let $G=\{a, e\}$ be the cyclic group of order two and let $\Phi$ be a field. Let $\mathfrak{A}=\Phi(G)$ and let $P:{ }^{2} \Phi(G) \rightarrow \Phi(G)^{2}$ be given by the matrix

$$
\left[\begin{array}{ll}
e & e \\
e & a
\end{array}\right]
$$

Then $\mathscr{M}=\mathscr{M}(\mathfrak{H} ; 2,2 ; P)$ is the (contracted) algebra of $S=\mathscr{M}^{0}(G ; 2,2 ; P)$ over $\Phi$.

(I) $\Phi$ has characteristic 2 . Then $\mathfrak{A}$ has a unique irreducible representation $\gamma: \mathfrak{A} \rightarrow \Phi$ defined by

$$
\gamma(\lambda e+\mu a)=\lambda+\mu .
$$


The kernel of $\gamma$ is

$$
M=\{\lambda e+\mu a \in \mathfrak{A}: \lambda+\mu=0\}=\left\{x \in \mathfrak{U}: x^{2}=0\right\} ;
$$

since $\mathfrak{A}$ has a unique maximal ideal, this is also $\operatorname{Rad} \mathfrak{A}$.

By Theorem 2.5,

$$
\operatorname{Rad} \mathscr{M}=B(M)=\left\{\left[\begin{array}{ll}
x & y \\
u & v
\end{array}\right] \in \mathscr{M}:(x+y+u+v)^{2}=0\right\}
$$

and, since rank $\gamma(P)=1$,

$$
\mathscr{H} / \operatorname{Rad} \mathscr{M} \approx \Phi \approx \mathfrak{A} / \operatorname{Rad} \mathfrak{A} .
$$

(II) $\Phi$ does not have characteristic two. $\mathfrak{A}$ has two irreducible representations, both of degree one, defined by

$$
\gamma_{1}(\lambda e+\mu a)=\lambda+\mu, \gamma_{2}(\lambda e+\mu a)=\lambda-\mu .
$$

Here $\mathfrak{A}$ is semisimple and is isomorphic to $\Phi \oplus \Phi$.

By Theorem 3.6,

$$
\operatorname{Rad} \mathscr{M}=B(0)=\left\{\left[\begin{array}{ll}
x_{1} & x_{2} \\
x_{3} & x_{4}
\end{array}\right] \in \mathscr{M}: x_{i}=\lambda_{i}(a+e), \lambda_{i} \varepsilon \Phi \text { and } \sum_{1}^{4} \lambda_{i}=0\right\} \text {. }
$$

Since rank $\gamma_{1}(P)=1$ and $\operatorname{rank} \gamma_{2}(P)=2$,

$$
\mathscr{M} / \operatorname{Rad} \mathscr{M} \approx(\Phi) \oplus(\Phi)_{2} \neq(\mathfrak{A} / \operatorname{Rad} \mathfrak{Q})_{t}
$$

for any positive integer $t$.

REMARK 2.7. Suppose that $\mathscr{M}=\mathscr{M}(\mathfrak{A} ; m, m ; P)$ and let $\gamma: \mathfrak{A} \rightarrow \operatorname{Hom}(A, A)$ be a morphism of $\mathfrak{A}$ onto the endomorphism ring of a finite dimensional vector space $A$ over a division ring $D$. Then we can give a short proof of Theorem 2.3, for this case, as follows. Let $C={ }^{n} A / \operatorname{Ker} \gamma(P)$ and let $Q R$ be the obvious natural factorisation of $\gamma(P)$ into $Q:{ }^{n} A \rightarrow C$ and $R: C \rightarrow A^{m}$. Then $C$ is finite dimensional so that $Q$ is projective and $R$ is injective. Hence

$$
R \gamma(\mathscr{M}) Q=R \operatorname{Hom}\left(A^{m},{ }^{n} A\right) Q=\operatorname{Hom}(C, C) .
$$

\section{A special case}

Definition 3.1. Let $\mathfrak{A}$ be a ring with identity and let $P$ be an $n \times m$ matrix over $\mathfrak{A}$. Then the invertibility rank of $P$ is the largest non-negative integer $s$ for which $P$ has an invertible $s \times s$ submatrix. (We adopt the convention that an empty submatrix is $0 \times 0$ ).

Definition 3.2. Let $\mathfrak{A}$ be a ring with identity and let $P$ be an $n \times m$ matrix over $\mathfrak{U}$, with invertibility rank $t$. Let $A$ and $B$ be permutation matrices over $\mathfrak{A}$ (cf. 7 , page 34 ) such that 


$$
A P B=\left[\begin{array}{ll}
M & P_{12} \\
P_{21} & P_{22}
\end{array}\right]
$$

where $M$ is an invertible $t \times t$ submatrix of $P$ and let

$$
Q=B\left[\begin{array}{ll}
M^{-1} & 0 \\
0 & 0
\end{array}\right] A .
$$

Then $P$ is suitable if $P Q P-P$ is over $\operatorname{Rad} \mathfrak{A}$.

Definitions 3.1 and 3.2 are slight generalisations of concepts introduced by Lallement and Petrich [7] for matrices over an algebra $\mathfrak{A}$, over a field $\Phi$; in their terminology, a suitable matrix is a $\Phi$-matrix.

Lemma 3.3, which follows, is a generalisation of [7], Lemma 3.3. The argument given in [7] serves to prove the present result if we note that any finite matrix over a division ring, is suitable.

LEMMA 3.3. Let $\mathfrak{A}$ be a ring with identity, which obeys the d.c.c. on right ideals, and let $P$ be an $n \times m$ matrix over $\mathfrak{A}$, with invertibility rank $t$. Then $P$ is suitable if and only if

$$
M \text {-rank } P=\text { t degree } \mathfrak{U} / M
$$

for each maximal ideal $M$ of $\mathfrak{A}$.

THEOREM 3.4. Let $\mathfrak{A}$ be a ring with identity which obeys the d.c.c. on right ideals. Let $\mathscr{M}=\mathscr{M}(\mathfrak{A} ; m, n ; P)$ be a Munn ring and suppose that $P$ has invertibility rank $t$. Then

$$
\mathscr{M} / \operatorname{Rad} \mathscr{M} \approx(\mathfrak{U} / \operatorname{Rad} \mathfrak{U})_{t}
$$

if and only if $P$ is suitable.

Proof. Suppose that $P$ is suitable and that

$$
\mathfrak{U} / \operatorname{Rad} \mathfrak{U} \approx\left(D_{1}\right)_{s_{1}} \oplus \cdots \oplus\left(D_{k}\right)_{s_{k}} .
$$

Then, by Lemma 3.3 and Theorem 2.5,

But

$$
\mathfrak{M} / \operatorname{Rad} \mathfrak{M} \approx\left(D_{1}\right)_{t s_{1}} \oplus \cdots \oplus\left(D_{k}\right)_{t s_{k}} .
$$

hence

$$
\left(D_{1}\right)_{t s_{1}} \oplus \cdots \oplus\left(D_{k}\right)_{t s_{k}} \approx\left(\left(D_{1}\right)_{s_{1}} \oplus \cdots \oplus\left(D_{k}\right)_{s_{k}}\right)_{t}
$$

as claimed.

$$
\mathscr{H} / \operatorname{Rad} \approx(\mathfrak{U} / \operatorname{Rad} \mathfrak{A})_{t}
$$

Conversely, let $M_{1}, \cdots, M_{k}$ be the distinct maximal ideals of $\mathfrak{U}$ and suppose that

$$
\mathfrak{A} / M_{i} \approx\left(D_{i}\right)_{s_{i}} \quad i=1,2, \cdots, k .
$$

Then, for each $i=1,2, \cdots, k$,

$$
\mathfrak{M} / B\left(M_{i}\right) \approx\left(D_{i}\right)_{t_{i}}
$$


Consider the isomorphism

$$
\left(D_{1}\right)_{s_{1} t} \oplus \cdots \oplus\left(D_{k}\right)_{s_{k} t} \approx\left(D_{1}\right)_{t} \oplus \cdots \oplus\left(D_{k}\right)_{t_{k}} .
$$

Then, because of the uniqueness of representation of simisimple rings, those summands on the left which are matrix rings over $D_{1}$ must be isomorphic to those on the right which are matrix rings over $D_{1}$. Suppose that $D_{1}, \cdots, D_{r}$ are isomorphic to one another but not to any of $D_{r+1}, \cdots, D_{k}$. Then

$$
\left(D_{1}\right)_{s_{1} t} \oplus \cdots \oplus\left(D_{r}\right)_{s_{r} t} \approx\left(D_{1}\right)_{t_{1}} \oplus \cdots \oplus\left(D_{r}\right)_{t_{r}}
$$

as rings and hence as vector spaces over $D_{1}$. Thus

$$
s_{1} t+\cdots+s_{r} t=t_{1}+\cdots+t_{r} .
$$

Further, since $P$ has an invertible $t \times t$ submatrix, $M_{i}$-rank $P \geqq s_{i} t, l \leqq i \leqq r$. Hence $s_{i} t=t_{i}, l \leqq i \leqq r$. Similarly $s_{i} t=t_{i}, r \leqq i \leqq k$ and so, by Lemma 3.3, $P$ is suitable.

Remark 3.5. Lallement and Petrich [7], Theorem 3.6, proved that, if $\mathscr{M}=$ $\mathscr{M}(\Phi(G) ; m, n ; P)$ is the algebra of a finite 0-simple semigroup $\mathscr{M}^{0}(G ; m, n ; P)$ over a field $\Phi$, then

$$
\mathscr{M} / \operatorname{Rad} \mathscr{M} \approx(\Phi(G) / \operatorname{Rad} \Phi(G))_{t}
$$

provided that $P$ is suitable with invertibility rank $t$. They have also proved the converse in the case when $\Phi$ is algebraically closed.

Although Theorem 3.4 gives a sufficient condition for the existence of an isomorphism

$$
\mathscr{M} / \operatorname{Rad} \mathscr{M} \approx(\mathfrak{U} / \operatorname{Rad} \mathfrak{U})_{t},
$$

the condition contained in the theorem is not necessary; it is possible for such an isomorphism to exist without $t$ being the invertibility rank of $P$.

EXAmple 3.6. Let $\Phi$ be a field and let $\mathfrak{A}=\Phi \oplus \Phi \oplus \Phi$. Then $\mathfrak{A}$ is the contracted algebra of $\{e, a, b, 0\}$ where

$$
e=(1,1,1) \quad a=(1,0,0) \quad b=(0,1,0) .
$$

Let $P:{ }^{3} \mathfrak{A} \rightarrow \mathfrak{A}^{3}$ be given by the matrix

$$
\left[\begin{array}{lll}
e & 0 & 0 \\
0 & a & 0 \\
0 & 0 & b
\end{array}\right]
$$

Then, since neither a nor $b$ is invertible, $P$ has invertibility rank 1 .

On the other hand, $\mathfrak{A}$ has three irreducible representations, each of degree one; these act as the projections $\mathfrak{U} \rightarrow \Phi$. If $\gamma$ denotes any one of these, then $\gamma(P)$ has rank 2 , over $\Phi$. Hence, if $\mathscr{M}=\mathscr{M}(\mathfrak{H} ; 3,3 ; P)$. 


$$
\mathscr{M} / \operatorname{Rad} \mathscr{M} \approx(\mathfrak{H})_{2} \approx(\mathfrak{U} / \operatorname{Rad} \mathfrak{A})_{2} ;
$$

the latter isomorphism holds since $\mathfrak{A}$ is semisimple.

Although Theorem 3.4 gives, modulo $\mathfrak{A}$, a complete description of $\mathscr{M} / \operatorname{Rad} \mathscr{M}$ when $P$ is suitable, it does not give much information about $\mathscr{M}$ itself. If we strengthen the conditions, in Definition 3.2, slightly, it is possible to obtain much more information about $\mathscr{M}$.

Definition 3.7. Let $\mathfrak{A}$ be a ring with identity and let $P$ be an $n \times m$ matrix over $\mathfrak{A}$, with invertibility rank $t$. Then $P$ is apt if there exist permutation matrices $A, B$ and a $t \times t$ invertible submatrix $M$ of $P$ such that

and $P=P Q P$ where

$$
A P B=\left[\begin{array}{ll}
M & P_{12} \\
P_{21} & P_{22}
\end{array}\right]
$$

$$
Q=\left[\begin{array}{ll}
M^{-1} & 0 \\
0 & 0
\end{array}\right]
$$

3.8 Remarks. If $\mathfrak{A}$ is semisimple, the concepts of aptness and suitability coincide.

If $\mathfrak{A}$ is an algebra over a field $\Phi$, then any finite matrix over $\mathfrak{A}$, whose entries are scalar multiplies of the identity, is apt; such a matrix can be regarded as a matrix over $\Phi$.

Any non-zero, apt matrix has an invertible entry.

An analog of Theorem 3.4 holds for Munn rings if we replace the word suitable by the word apt and the radical of $\mathscr{M}$ by the basic radical $B(0)$ of $\mathscr{M}$; we shall write the latter as $B(\mathscr{M})$. In order to give a compact proof of this analog, we prove the following general proposition about Munn rings.

PROPOSITION 3.9. Let $P$ be an $n \times m$ matrix over a ring with identity and let $A, B$ be permutation matrices such that

$$
A P B=\left[\begin{array}{ll}
M & P_{12} \\
P_{21} & P_{22}
\end{array}\right]
$$

where $M$ is an invertible $t \times t$ submatrix of $P$; let

$$
F=\left[\begin{array}{cc}
I_{t} & 0 \\
0 & D
\end{array}\right]
$$

where $D=P_{22}-P_{21} M^{-1} P_{12}$. Then there is an isomorphism between the Munn rings $\mathscr{M}(\mathfrak{U} ; m, n ; P)$ and $\mathscr{M}(\mathfrak{U} ; m, n ; F)$.

Proof. Let

$$
U=A^{-1}\left[\begin{array}{ll}
M & 0 \\
P_{21} & I_{n-t}
\end{array}\right] \quad \text { and } V=\left[\begin{array}{ll}
I_{t} & M^{-1} P_{12} \\
0 & I_{m-t}
\end{array}\right] B^{-1}
$$

Then $U$ and $V$ are invertible and $P=U F V$. 
Define $\phi: \mathscr{M}(\mathfrak{A} ; m, n ; P) \rightarrow \mathscr{M}(\mathfrak{A} ; m, n ; F)$ by

$$
X \phi=V X U \text { for each } X \in \mathscr{M} \text {. }
$$

Then, since $U, V$ are invertible, $\phi$ is an isomorphism of the additive structures. Further, since $P=U F V, \phi$ preserves multiplication and thus is a ring isomorphism.

CoROllaRy 3.10. Let $P$ be an apt $n \times m$ matrix over a ring $\mathfrak{A}$, with identity, with invertibility rank $t$. Then

where E has matrix

$$
\mathscr{H}(\mathfrak{A} ; m, n ; P) \approx \mathscr{M}(\mathfrak{A} ; m, n ; E)
$$

$$
\left[\begin{array}{ll}
I_{t} & 0 \\
0 & 0
\end{array}\right] \text {. }
$$

Proof. Let $A, B, M$ be such that $P Q P=P$; then simple matrix calculation shows that $D=0,(D$ as in Proposition 3.9). The result is now immediate from the proposition.

TheOREM 3.11. Let $\mathscr{M}=\mathscr{M}(\mathfrak{U} ; m, n ; P)$ be a Munn ring where $P$ has invertibility rank $t$ and $\mathfrak{Q}$ is an algebra of finite degree over a field $\Phi$. Then

if and only if $P$ is apt.

$$
\mathscr{M} / B(\mathscr{M}) \approx(\mathfrak{U})_{t}
$$

Proof. If $P$ is apt then, by Corollary 3.10 , we may suppose $\mathscr{M}=\mathscr{M}(\mathfrak{A} ; m, n ; E)$. It is an easy matter to show that $B(\mathscr{H})$ consists of all

$$
\left[\begin{array}{ll}
X_{11} & X_{12} \\
X_{21} & X_{22}
\end{array}\right]
$$

in $\mathscr{M}$ with $X_{11}=O_{t}$, the zero $t \times t$ matrix over $\mathfrak{U}$. Hence, clearly $\mathscr{M} / B(\mathscr{M}) \approx(\mathfrak{A})_{t}$.

Conversely, we can suppose $\mathscr{M}=\mathscr{M}(\mathfrak{A} ; m, n ; F)$ where $F$ is as in Proposition 5.9 and $M$ is any $t \times t$ invertible submatrix of $P$. Then, for

$$
\begin{gathered}
X=\left[\begin{array}{ll}
X_{11} & X_{12} \\
X_{21} & X_{22}
\end{array}\right] \\
F X F=\left[\begin{array}{ll}
X_{11} & X_{12} D \\
D X_{21} & D X_{22} D
\end{array}\right]
\end{gathered}
$$

and so, if $X \in B(\mathscr{M}), X_{11}=0$. Thus

$$
\operatorname{dim} B(\mathscr{H}) \leqq\left(m n-t^{2}\right) \text { degree } \mathfrak{A} \text {. }
$$

But $\operatorname{dim} B(\mathscr{M})=m n$ degree $\mathfrak{A}-t^{2}$ degree $\mathfrak{A}$, by hypothesis. Hence

$$
B(M)=\left\{X \in \mathscr{M}: X_{11}=0\right\} \text {. }
$$


Let $X_{12}$ be the $t \times(m-t)$ matrix given by

$$
\left(X_{12}\right)_{i j}= \begin{cases}e & \text { if } i=i^{\prime}, j=j^{\prime}, 1 \leqq i \leqq t, 1 \leqq j \leqq m-t \\ 0 & \text { otherwise }\end{cases}
$$

Then

$$
X=\left[\begin{array}{ll}
0 & X_{12} \\
0 & 0
\end{array}\right] \in B(\mathfrak{M}) \quad \text { and so } X_{12} D=0 .
$$

But $X_{12} D$ has $i^{\prime}$-th row the $j^{\prime}$-th row of $D$. As $i^{\prime}, j^{\prime}$ range over $1 \leqq i^{\prime} \leqq t$, $i \leqq j^{\prime} \leqq m-t$, this shows that all rows of $D$ are zero and hence $D=0$. This means precisely that $P$ is apt.

CoRollary 3.12. (To the proof.) Let $P$ be an apt $n \times m$ matrix over an algebra $\mathfrak{A}$ of finite degree over a field $\Phi$, with invertibility rank $t$. Then, if $A, B$ are permutation matrices over $\mathfrak{A}$ such that

$$
A P B=\left[\begin{array}{ll}
M & P_{12} \\
P_{21} & P_{22}
\end{array}\right]
$$

where $M$ is $t \times t$ and invertible, $P Q P=P$ where

$$
Q=B\left[\begin{array}{ll}
M^{-1} & 0 \\
0 & 0
\end{array}\right] A .
$$

\section{Finite semigroups}

In this section we apply the theory obtained in previous sections to the algebra of a finite semigroup. As a first step we state the following theorem which characterises the algebra of a finite 0 -simple semigroup. Because this algebra is a Munn ring the result is an immediate corollary to Theorem 2.5.

TheOREM 4.1. Let $S \approx \mathscr{M}^{0}(G ; m, n ; P)$ be a finite 0 -simple semigroup and let $\Phi$ be a field. Then the (contracted) algebra $\Phi(S)$ of $S$ over $\Phi$ is isomorphic to the Munn ring $\mathscr{M}=\mathscr{M}(\Phi(G) ; m, n ; P)$.

Suppose that

$$
\Phi(G) / \operatorname{Rad} \Phi(G) \approx\left(D_{1}\right)_{s_{1}} \oplus \cdots \oplus\left(D_{r}\right)_{s_{r}}
$$

where $D_{i}, 1 \leqq i \leqq r$ is a division ring. For each $i$, let

$$
M_{i}=\left(D_{1}\right)_{s_{1}} \oplus \cdots \oplus\left(D_{i-1}\right)_{s_{i-1}} \oplus\left(D_{i+1}\right)_{s_{i+1}} \oplus \cdots \oplus\left(D_{r}\right)_{s_{r}}
$$

and let $t_{i}=M_{i}$-rank $P$. Then

and

$$
\operatorname{Rad} \Phi(S)=\{X \in \mathscr{M}: P X P \text { is over } \operatorname{Rad} \Phi(G)\}
$$

$$
\Phi(S) / \operatorname{Rad} \Phi(S) \approx\left(D_{1}\right)_{t_{1}} \oplus \cdots \oplus\left(D_{r}\right)_{t_{r}} .
$$


Munn [11], has shown that, if $S$ is a regular rectangular semigroup with zero adjoined, $\Phi(S) / \operatorname{Rad} \Phi(S) \approx \Phi(G) / \operatorname{Rad} \Phi(G)$, where $G$ is a non-zero maximal subgroup of $S$ and $\Phi$ is any field. Example 2.6 shows that the converse is not true; the semigroup $S$ considered there is not a regular rectangular semigroup with zero adjoined. In order to characterise these semigroups among all finite 0 -simple semigroups, we use Theorem 3.11.

THEOREM 4.2. Let $S$ be a finite 0 -simple semigroup and let $G$ be a non-zero maximal subgroup of $S$. Let $\Phi$ be a field. Then $S$ is a regular rectangular semigroup if and only if

$$
\Phi(S) / B(\Phi(S)) \approx \Phi(G) .
$$

Proof. Since $S$ is completely 0 -simple, we may assume that $p_{11}=e$, the identity of $G$ and that $p_{\lambda i} \in\{e, 0\}$ if $i=1$ or $\lambda=1$; [5], page 95 . Then, by the result of Thierrin, [14], which is given in [5], page 98. Example $8 \mathrm{~b}, S$ is a regular rectangular semigroup with zero adjoined if and only if $p_{\lambda i}=e$ for each $i \in I, \lambda \in \Lambda$.

If each entry in $P$ is $e$ then, clearly, $P$ is apt with invertibility rank one. Hence, by Theorem 3.11, $\mathscr{M} / B(\mathscr{M}) \approx \Phi(G)$ where $\mathscr{M}=\mathscr{M}(\Phi(G) ; m, n ; P) \approx \Phi(S)$.

Conversely, if $\mathscr{M}_{/} B(\mathscr{M}) \approx \Phi(G)$, then $P$ must have invertibility rank 1 and so is apt. Hence, if we partition $P$ as

$$
\left[\begin{array}{ll}
e & P_{12} \\
P_{21} & P_{22}
\end{array}\right],
$$

$P_{22}=P_{21} P_{12}$. Since each row and column of $P$ has an invertible entry, this implies that no entry in $P_{21}$ or $P_{12}$ is zero; hence each is $e$. Therefore each entry in $P_{22}$, and thus in $P$, is $e$. This shows that $S$ is a regular rectangular semigroup with zero adjoined.

Definition 4.3. A principal series for a finite semigroup $S=S^{0}$ is a series

$$
S \supset S_{0} \supset S_{1} \cdots \supset S_{n}=\{0\}
$$

of ideals of $S$ such that no factor $S_{i} / S_{i+1}, 0 \leqq i \leqq n$, has a proper ideal.

Clearly, any finite semigroup has a principal series. Green [6] has proved that any two principal series for $S$ have the same length and that the factors are pairwise isomorphic. Further each principal factor ([5], page 72) of $S$ appears exactly once in each principal series. The factors in a principal series are either null semigroups of order two or are completely 0 -simple. The completely 0 -simple factors are called the regular factors of $S$.

THEOREM 4.4. Let $S$ be a finite semigroup and let $J_{1}, \cdots, J_{r}$ be the regular principal factors of $S$. Let $\Phi$ be a field. Then

$$
\Phi(S) / \operatorname{Rad} \Phi(S) \approx\left(\Phi\left(J_{1}\right) / \operatorname{Rad} \Phi\left(J_{1}\right)\right) \oplus \cdots \oplus\left(\Phi\left(J_{r}\right) / \operatorname{Rad} \Phi\left(J_{r}\right)\right) .
$$

Proof. We use the terminology of [5], Theorem 5.33 which is due to Munn 
[12]. According to that theorem, the apex of an irreducible representation $\gamma$ of $S$ is a regular principal factor $J$ of $S$ and there is an irreducible representation $\gamma^{\prime}$ of $J$ such that

$$
\gamma(x)=\gamma^{\prime}(\bar{x} \bar{e})
$$

for each $x \in \Phi(S)$ where $\bar{e}$ is such that $\operatorname{Rad} \Phi(J)+\bar{e}$ is the identity of $\Phi(J) /$ $\operatorname{Rad} \Phi(J)$. It follows that

$$
\gamma(\Phi(S))=\gamma^{\prime}(\Phi(J))
$$

Conversely, given any irreducible representation $\gamma^{\prime}$ of a regular principal factor $J$ of $S, \gamma$ defined by (4.1) is an irreducible representation of $S$. Hence, since $\Phi(S) /$ $\operatorname{Rad} \Phi(S)$ is isomorphic to the direct sum of its simple homomorphic images

$$
\Phi(S) / \operatorname{Rad} \Phi(S) \approx\left(\Phi\left(J_{1}\right) / \operatorname{Rad} \Phi\left(J_{1}\right)\right) \oplus \cdots \oplus\left(\Phi\left(J_{r}\right) / \operatorname{Rad} \Phi\left(J_{r}\right)\right)
$$

as claimed.

If we combine Theorems 4.1, 4.4, we have a computational method for determining the algebra of a finite semigroup $S$ modulo its radical. The determination of the radical in the general case remains an open question although the radical can be determined if $S$ is commutative or has just one regular $\mathscr{D}$-class. (cf. [5], Theorem 5.31 for $S$ commutative).

\section{References}

[1] W. P. Brown, 'Generalised matrix algebras', Canadian J. Math. 7 (1955), 188-190.

[2] A. H. Clifford, 'Matrix representations of completely simple semigroups', Amer. J. Math. 64 (1942), 327-342.

[3] A. H. Clifford, 'Basic representations of completely simple semigroups', Amer. J. Math. 82 (1960), $430-434$.

[4] A. H. Clifford and G. B. Preston, The algebraic theory of semigroups, Vol. 1 (Math. Survey 7, Amer. Math. Soc., 1961)

[5] J. A. Green, 'On the structure of semigroups', Annals of Math. 54 (1951), 163-172.

[6] N. Jacobson, The structure of rings (American Math. Soc. Colloquium Publications, Vol. 37, 1956).

[7] G. Lallement and M. Petrich, 'Irreducible matrix representations of finite semigroups', Trans. Amer. Math. Soc. 139 (1969), $393-412$.

[8] D. B. McAlister, 'Basic representations of arbitrary semigroups', Trans. Amer. Math. Soc. 135 (1969), 311-342.

[9] D. B. McAlister, 'The category of representations of a completely 0-simple semigroup', J. Austr. Math. Soc. 2 (1971), 193-210.

[10] N. H. McCoy, The theory of rings (Macmillan, New York, 1964).

[11] W. D. Munn, 'On semigroup algebras', Proc. Camb. Phil. Soc. 51 (1955), 1-15.

[12] W. D. Munn, 'Irreducible matrix representations of semigroups', Quarterly Jour. Math. Oxford, Ser. (2), 11 (1960), 295-309.

[13] M. P. Schutzenberger, ' $\mathscr{D}$ représentation des demigroupes', C. R. Acad. Sci. Paris. 224 (1957) 1994-96.

[14] G. Theirrin, 'Demigroupes inversés et rectangulaires', Bull. Roy. Belg. Bull. Cl. Sci. (5), 41 (1955), 83-92.

\section{Department of Pure Mathematics}

Queen's University, Belfast 